\title{
Development and evaluation of a matheuristic for the combined beam angle and dose distribution problem in radiotherapy planning
}

\author{
Thalita Monteiro Obal, Chandra Ade Irawan, Dylan Jones, Djamila Ouelhadj, Helenice \\ Oliveira Florentino, Vania Gryczak, Neida Maria Patias Volpi, Volmir Eugenio Wilhelm
}

[Received on 19 November 2018]

\begin{abstract}
Radiotherapy planning is a vital for ensuring the maximum level of effectiveness of treatment. In the planning task, there are at least two connected decision problems that can be modelled and solved using Operational Research techniques: determining the best position of the radiotherapy machine (beam angle problem) and the optimal dose delivered through each beam (dose distribution problem). This paper presents a mathematical optimisation model for solving the combined beam angle and dose distribution problems in the presence of multiple objectives. A matheuristic based on Tabu Search (called TSrad) is developed to solve realistic large-scale instances. The performance of the proposed method is assessed on two prostate cancer instances, namely a single computed tomography (CT) slice and a set of CT slices (3D problem). For the single-slice problem, the results of TSrad are compared to the optimal solutions obtained by an exact method. Our experiments show that TSrad is able to achieve optimality for some instances. For the multi-slice problem, our experiments show that TSrad produces viable solutions that can be attained in a reasonable computational time.
\end{abstract}

Keywords: Healthcare; Radiotherapy optimisation; Tabu Search; Matheuristic.

\section{Introduction}

Radiotherapy is currently one of the main modalities in treating cancer. A critical factor for effective radiotherapy planning is to determine the necessary dose to reduce or eliminate a tumour and to ensure that the side effects on the surrounding healthy organs are minimised. The classical problem in radiotherapy planning includes two important decisions, as follows (Ehrgott et al., 2008):

- The position of the gantry (the beam angle/direction problem).

- The quantity of the dose that needs to be delivered through each beam and sub-beam (the dose distribution problem).

An optimisation model for the dose distribution problem was first introduced by Bahr et al. (1968). Thereafter, many improvements to the modelling process and solution methodologies have been conducted resulting in potentially better radiotherapy planning. Romeijn et al. (2006), Acosta et al. (2009), Viana et al. (2011) and Obal et al. (2013) proposed the solution of the dose distribution problem using an exact methodology. Rocha et al. (2013), Dias et al. (2014) and Dias et al. (2015) developed non-exact approaches to solve the beam direction problem. Considering the inherent characteristics of radiotherapy planning problems, some research has been conducted to solve both beam angle and dose distribution problems as the dose distribution depends on the beam angle. For example, Souza (2004), Lim et al. (2008), Lim et al. (2012) and Gevert et al. (2013) used exact methods, whilst Bertsimas et al. (2013), Breedveld (2013) and Voet (2014) used hybrid approaches to solve the problems. 
Obal et al. (2015) proposed a matheuristic method, a hybridization of an exact method and a Genetic Algorithm (GA), and applied it to a prostate cancer case with one CT slice. Petrovic et al. (2016) used a case based reasoning system (CBR) where a treatment plan for a new patient is retrieved from a case base containing patient cases treated in the past and their treatment plans. They also investigated an approach called knowledge-light adaptation to avoid too much requirement of domain knowledge. A two phase strategy for a multiobjective problem was proposed by Cabrera et al. (2018a), where beam angles are selected by a deterministic local search algorithm and dose distribution is calculated using an exact nonlinear programming algorithm. Cabrera et al. (2018b) develop two bi-objective local search algorithms which aim to find a set of (approximately) efficient beam angle configurations.

This paper deals with the IMRT (Intensity Modulated Radiotherapy) combined beam angle and dose distribution problems where the number of beams that can be selected from the available ones is known. The problem is inherently multi-objective because there is a conflict between the goals of delivering a sufficiently high dosage to the tumour voxels and delivering a sufficiently low (and ideally zero) dosage to the healthy tissue and organs at risk. Here, we formulate the IMRT problem as a Goal Programming (GP) model that can be solved using exact methods, heuristics or metaheuristics. In this case, a matheuristic base on Tabu Search (TSrad) is proposed to solve the GP model as the exact method experienced difficulties when solving relatively large problems (the realistic 3D instances). Matheuristic is a hybridization of a metaheuristic and an exact method that can be used for finding good solutions (Maniezzo et al., 2010). To the best of our knowledge, the proposed matheuristic method has not been reported in the literature to address the combined beam angle and dose distribution problems. In this present research work, the main contributions are as follows:

- The development of a novel multi-objective optimisation model using goal programming for solving the combined beam angle and dose distribution problem.

- The development of a novel matheuristic (TSrad) for solving the model.

- The generation of optimal solutions for one CT slice instances along with viable solutions for more challenging $3 \mathrm{D}$ problems.

The remainder of this paper is organized as follows: the mathematical formulation of the multiobjective radiotherapy planning problem is given in Section 2. The proposed matheuristic is described in Section 3. Section 4 presents the computational results of the proposed methods. The final section provides a summary of the findings.

\section{Multi-objective optimisation model}

This section presents the multi-objective optimisation model for the combined beam angle and dose distribution problem. In this study, Goal Programming (GP) is used to model the problem due to the inherent goal-based nature of the objectives. Furthermore, the pragmatic nature of the goal programming model allows solutions to be found to computationally complex multi-objective models, without imposing an excessive extra computational burden on account of the multiple objectives. The solutions to a goal programming model are optimal with respect to the preferences of the decision maker that are inputted to the model as target values and weights associated with unwanted deviational variables. Thus goal programming is an a priori multi-objective technique. Providing the target values are sufficiently strictly set then the resulting solution is Pareto optimal with respect to the underlying multiple objectives of the problem (Jones and Tamiz, 2010). In our generic model the preferences of the decision maker are set by the weights which are elicited a priori. 
In the model, a set of available beams $(B)$ is given where the optimal beam configuration consisting of $\eta$ beams needs to be determined and $\eta<<|B|$. Once a set of selected beams has been determined, the model will define their dose distribution. We present a mathematical model using mixed integer linear programming (MILP) for such problem. The following notations are used to describe the sets and parameters of the proposed model.

Sets

$S$ : set of CT slices indexed by $s$.

$V$ : set of voxels indexed by $v$ with $\rho=|V|=l \times c \times|S|$

$\hat{V}_{s}:$ set of voxels for slice $s \in S$

$V_{s}^{R}$ : set of risk voxels for slice $s \in S\left(V_{s}^{R} \subset \hat{V}_{s} \subset V\right)$

$V_{s}^{H}$ : set of healthy voxels for slice $s \in S\left(V_{s}^{H} \subset \hat{V}_{s} \subset V\right)$

$V_{s}^{T}$ : set of tumour voxels for slice $s \in S\left(V_{s}^{T} \subset \hat{V}_{s} \subset V\right)$

$B$ : set of possible beam angles with $b$ as its index

$Q_{b}$ : set of sub-beams of beam $b(b \in B)$ indexed by $q$.

$|\eta|$ : the number of beams that can be selected.

$r$ : set of organs at risk.

Parameters

$d^{R}$ : the upper limit dose allowed to reach the at risk voxels.

$d^{H}$ : the upper limit dose allowed to reach the healthy voxels.

$d^{T}$ : the dose intensity prescribed by the doctor to address the tumour voxels.

$a_{b q s v}$ : the absorption percentage of the of the dose emitted from sub-beam $q$ of beam $b$ for slice $s$ on voxel $v$.

Decision variables

$K_{b q s}:$ the quantity of the dose delivered by sub-beam $q$ of beam $b$ for slice $s$.

$L_{b}:=1$ if beam $b$ is selected, or $=0$ otherwise.

$n_{s v}^{R}$ : the negative deviation between the absorption dose and the recommended upper limit for slice $s \in S$ on voxel $v$ in the organ at risk $\left(v \in V_{s}^{R}\right)$.

$p_{s v}^{R}$ : the positive deviation between the absorption dose and the recommended upper limit for slice $s \in S$ on voxel $v$ in the organ at risk $\left(v \in V_{s}^{R}\right)$.

$n_{s v}^{H}$ : the negative deviation between the absorption dose and the recommended upper limit for slice $s \in S$ on voxel $v$ in the healthy organ $\left(v \in V_{s}^{H}\right)$. 
$p_{s v}^{H}$ : the positive deviation between the absorption dose and the recommended upper limit for slice $s \in S$ on voxel $v$ in the healthy organ $\left(v \in V_{s}^{H}\right)$.

$n_{s v}^{T}$ : the negative deviation between the absorption dose and the recommended upper limit for slice $s \in S$ on voxel $v$ in the tumour $\left(v \in V_{s}^{T}\right)$.

$p_{s v}^{T}$ : the positive deviation between the absorption dose and the recommended upper limit for slice $s \in S$ on voxel $v$ in the tumour $\left(v \in V_{s}^{T}\right)$.

\section{Objective functions}

The problem is treated as a multi-objective problem with the presence of four objectives as follows:

- Minimise total unwanted positive deviations of the organ at risk voxels $\left(f_{1 r^{j}}\right)$

$$
\text { Minimise } f_{1 r^{j}}=\sum_{s \in S} \sum_{v \in V_{s}^{R}}\left(p_{s v}^{R}\right) \quad \forall r^{j} \in r
$$

- Minimise total unwanted positive deviations of the healthy voxels $\left(f_{2}\right)$

$$
\text { Minimise } f_{2}=\sum_{s \in S} \sum_{v \in V_{s}^{H}}\left(p_{s v}^{H}\right)
$$

- Minimise total unwanted positive deviations of the tumours voxels $\left(f_{3}\right)$

$$
\text { Minimise } f_{3}=\sum_{s \in S_{v}} \sum_{v}\left(p_{s v}^{T}\right)
$$

- Minimise total unwanted negative deviations of the tumours voxels $\left(f_{4}\right)$

$$
\text { Minimise } f_{4}=\sum_{s \in S} \sum_{v \in V_{s}^{T}}\left(n_{s v}^{T}\right)
$$

The model aims to find the optimal beam configuration along with the dose distribution for each beam so as the quantity of the dose absorbed by the tumour voxels is close to dose $d^{T}$, which is represented by objective functions (3) and (4). In addition, it also minimises the quantity of dose absorbed by the organ at risk and healthy structures, given by objective functions (1) and (2). The proposed model is formulated as a weighted GP model where the weights $\alpha, \beta, \gamma_{1}$ and $\gamma_{2}$ are introduced to represent the importance of $f_{1}, f_{2}, f_{3}$ and $f_{4}$, respectively. So, $\gamma_{1}$ and $\gamma_{2}$ are the weights related to the tumour organ, whereas $\alpha_{r^{\prime}}$ and $\beta$ are related to the organ at risk and healthy tissue, respectively. A single objective function (5) is proposed to minimise the value of $g$ which consist of objective functions (1)-(4). In the objective function (5), the normalisation process of the weights is not required as the deviational variables have the same measurement unit. 


$$
\min \quad Z=\left(\sum^{r} \alpha_{r^{\prime}} f_{1 r^{\prime}}+\beta f_{2}+\gamma_{1} f_{3}+\gamma_{2} f_{4}\right)
$$

subject to

$$
\left\{\begin{array}{cc}
\sum_{b \in B} \sum_{q \in Q_{b}}\left(a_{b q s v} \cdot K_{b q s}\right)+n_{s v}^{R}-p_{s v}^{R}=d^{R}, & \forall s \in S, v \in V_{s}^{R} \\
\sum_{b \in B} \sum_{q \in Q_{b}}\left(a_{b q s v} \cdot K_{b q s}\right)+n_{s v}^{H}-p_{s v}^{H}=d^{H} & \forall s \in S, v \in V_{s}^{H} \\
\sum_{b \in B} \sum_{q \in Q_{b}}\left(a_{b q s v} \cdot K_{b q s}\right)+n_{s v}^{T}-p_{s v}^{T}=d^{T} & \forall s \in S, v \in V_{s}^{T} \\
\sum_{b \in B} L_{b}=\eta & \\
K_{b q s} \leqslant M \cdot z b & \\
K_{b q s}, n_{s v}^{R}, p_{s v}^{R}, n_{s v}^{H}, p_{s v}^{H}, n_{s v}^{T}, p_{s v}^{T} \geqslant 0 & \\
L_{b}=\{0,1\} &
\end{array}\right.
$$

where $M$ is an arbitrarily large constant.

Constraints (6) and (7) ensure that the absorption of the dose emitted from all sub-beams on an organ at risk or healthy voxel is equal to the upper limit dose absorption allowed on this type of voxel along with its positive and negative deviations. Constraints (8) guarantee that the recommended dose with its deviation for a tumour voxel is the same with the quantity of the dose absorbed by the voxel. Constraint (9) ensures that $\eta$ beams will exactly be selected. Constraints (10) guarantee that the dose is only required for the selected beams. Constraints (11) state non-negative variables. In sign restrictions (12), variable $L_{b}$ is set as binary.

The proposed model is a mixed integer goal programme with $\left(2 .|V| \cdot|S|+|B| \cdot\left|Q_{b}\right| \cdot|S|+|B|\right)$ decision variables and $\left(|V| \cdot|S|+|B| \cdot\left|Q_{b}\right| \cdot|S|+1\right)$ constraints which is considered as a large-scale problem. In real 3D cases, for example, the number of decisions variables and constraints can be higher than 15 and 7 billion, respectively. With this size, it is currently impossible to solve the problem on a standard desktop computer using an exact method. Although a super computer could potentially be utilised for solving this problem, the aim of this study is to produce a methodology potentially usable in current hospitals, where the use of a supercomputer is not an option. Therefore, a matheuristic is proposed to overcome the computational complexity of exact methods and give near-optimal solutions in reasonable computational time on a standard desktop computer.

\section{Development of the Matheuristic}

In this section, a matheuristic is developed to solve the beam angle and dose distribution problem. Matheuristics is found to be successful in solving optimisation problems (Maniezzo et al., 2010; Irawan et al., 2017a, Irawan et al., 2017b). We refer to the proposed matheuristic as TSrad, where Tabu Search (TS) is implemented (Glover, 1989; Talbi, 2009). Tabu search is a metaheuristic search where this solution method can be applied to solve problems in health care (Djenic et al, 2017; Xiao et al. 2018). TSrad is applied to find the best beam configuration (beam angle problem) where $\eta$ beams from the $|B|$ possible beams available are selected. Within the search, an exact method is used to solve the dose distribution problem and hence to calculate the objective function value using the set of selected beams determined by the metaheuristic approach.

In case that a set of selected beams $X, X \in B,|X|=\eta$ are known/fixed, the problem reduces to the dose distribution problem whose the mathematical formulation is presented as follows. 


$$
\min \quad Z=\left(\alpha f_{1}+\beta f_{2}+\gamma_{1} f_{3}+\gamma_{2} f_{4}\right)
$$

subject to

$$
\left\{\begin{array}{cl}
\sum_{b \in B} \sum_{q \in Q_{b}}\left(a_{b q s v} \cdot K_{b q s}\right)+n_{s v}^{R}-p_{s v}^{R}=d^{R}, & \forall s \in S, v \in V_{s}^{R} \\
\sum_{b \in B} \sum_{q \in Q_{b}}\left(a_{b q s v} \cdot K_{b q s}\right)+n_{s v}^{H}-p_{s v}^{H}=d^{H} & \forall s \in S, v \in V_{s}^{H} \\
\sum_{b \in B} \sum_{q \in Q_{b}}\left(a_{b q s v} \cdot K_{b q s}\right)+n_{s v}^{T}-p_{s v}^{T}=d^{T} & \forall s \in S, v \in V_{s}^{T} \\
K_{b q s}, n_{s v}^{R}, p_{s v}^{R}, n_{s v}^{H}, p_{s v}^{H}, n_{s v}^{T}, p_{s v}^{T} \geqslant 0 &
\end{array}\right.
$$

Constraints (14)-(16) act similarly to Constraints (6)-(8) except that in this model, beam $b$ is element of the selected beams $(X)$ instead of all possible beams available $(B)$. This model is a linear programming with $\left(2 .|V| .|S|+|X| \cdot\left|Q_{b}\right| \cdot|S|\right)$ decision variables and $(|V|)$ constraints. The model is significantly easier to solve using an exact method as compared to the combined beam angle and dose distribution problem.

The main steps of the proposed TSrad are presented in Algorithm 3.1. Firstly, as an initial solution, $\eta$ beams are randomly selected from the set of possible beams $(B)$. Let $X$ be a set of solution whereas $\tilde{Y}$ a set of $|B|-\eta$ beams not selected, $X \cup \tilde{Y}=B$ and $X \cap \tilde{Y}=\emptyset$. The quality of the solution $(K)$ is evaluated by solving the dose distribution problem (Equations 13-17) using the exact method (CPLEX) which also determines the value of decision variable $K_{b q s}$. In this solution method, two parameters are used, namely the number of neighbourhood solutions $\left(n^{s}\right)$ and the size of tabu-list $t^{t}$. The procedure runs until the computing time, $t^{\max }$ is reached.

Algorithm 3.1 The main steps of TSrad

Function $\operatorname{TSrad}\left(B, \eta, n^{s}, t^{t}, t^{\max }\right)$

1. Generate randomly an initial solution $X$ and construct set $\tilde{Y}$.

2. Evaluate $X$ by solving the dose distribution problem using an exact method. Let $Z$ be its objective function value.

3. Set $X^{\prime}=X^{*}=X$ and $Z^{\prime}=Z^{*}=Z$ where $X^{*}$ denote the best solution to the current time.

4. Set tabu-list $=\emptyset$ and $N(X)=\emptyset$.

5. Repeat the following steps (a - f) until computational time $t^{\max }$ is reached:

(a) Construct the neighbourhood of $X, N(X)$, with $n^{s}$ solutions. Each neighbour solution $X_{k}^{N}, k=1, \ldots, n^{s}$ is generated by copying set $X$ and removing randomly a beam, say beam $\mathrm{i}$, from this copy $(i \in X)$ and inserting randomly a beam, say beam $j$, into this copy, where $j \in \tilde{Y}$. The $k^{t h}$ modified copy is the $k^{t h}$ neighbour solution of the $X, X_{k}^{N}$. Store $X_{k}^{N}$ in $N(X)$.

(b) Evaluate the solutions in $N(X)$ by solving the dose distribution problem using an exact method.

(c) Select the best solution $X^{\prime} \in N(X)$ where this solution is treated as 'candidate move'. 
(d) If $Z^{\prime}<Z^{*}$, set $X^{*}=X^{\prime}$ and $Z^{*}=Z^{\prime}$.

(e) While 'candidate move' is tabu and the aspiration criterion is not satisfied (i.e. $Z^{\prime}>$ 0.9.Z), select next best solution $X^{\prime} \in N(X)$ and mark as 'candidate move'.

(f) If (candidate move is not tabu) or (candidate move is tabu and the aspiration criteria is satisfied) then insert the move (beam $i$ and $j$ ) in the tabu-list and set $X=X^{\prime}$.

6. Choose $X^{*}$ as the best solution along with the dose distribution for each selected beam. The best objective function value will be $Z^{*}$.

\section{Computational experiments}

The data used for examining the performance of the proposed methods is cancer data relating to the prostate region. The prostate cancer was selected because, in general, it is one of the most common cases. The data is obtained from Erasto Gaertner Hospital (Curitiba/PR/Brazil), where the ethical committee has approved the project. The data is composed of two instances where the first instance consists of one CT slice data given in Figure 1, whereas the second one comprises a set of thirty CT slices (3D). The first instance can be considered as a small instance that may be solved optimally by an exact method using CPLEX. This instance is also solved using the matheuristic in order to assess its performance by comparing its results with the optimal solution. The second instance is a large scale instance which is a realistic radiotherapy planning configuration. For this instance, the optimal solutions cannot be generated using an exact method due the large size of the instance. Therefore, TSrad is used to solve such large practical instances.

In the experiments on both instances, 72 possible beam angles are used, where the angle of the beam corresponding to a perpendicular plan of the patient is set from $0^{\circ}$ to $355^{\circ}$ with increments of $5^{\circ}$ using a counter clock wise direction. Currently, it is common practice to use 4 or 6 beam directions in the prostate cancer radiotherapy treatment. Therefore, in the experiments $\eta$ is set to 4 and 6 for both instances. Based on the clinical practice, the dose limit for the healthy and the tumour structures are set to 60 and $70 \mathrm{~Gy}$, respectively. Three organs at risk are considered namely the bladder, head of femur, and rectum where their dose limits are set to 65,50 and $50 \mathrm{~Gy}$, respectively. The weights of the objective function (5) are set to 0.4 for $\gamma_{1}$ and $\gamma_{2}$ whereas the values of $\alpha$ and $\beta$ are set to 0.1 . These values are chosen based on the experiments conducted in Gevert et al. (2013), which show that this set of weight values provide better results for this case study. This can be considered as a conservative strategy to assign a large relative importance to preventing the damage of healthy and organ tissue.

Table 1 presents the upper limit percentage of region in the organ at risk that is allowed to receive a certain dose. For example, in the rectum column of Table 1 , V75 $<15 \%$ means that it is not recommended that more than $15 \%$ of rectum region receives more than $75 \mathrm{~Gy}$. Although this parameter is not directly considered by the proposed model, this data is important to determine the quality of the solutions generated for the treatment. It can be noted that in addition to having a small objective function value $(Z)$, a good solution needs to meet several criteria as follows: (i) for organ at risk, the upper limit percentage constraint presented in Table 1 and (ii) for the tumour structure, the percentage of the region that receives the dose and the average dose per voxel which is desirable to be close to the prescribed one. 


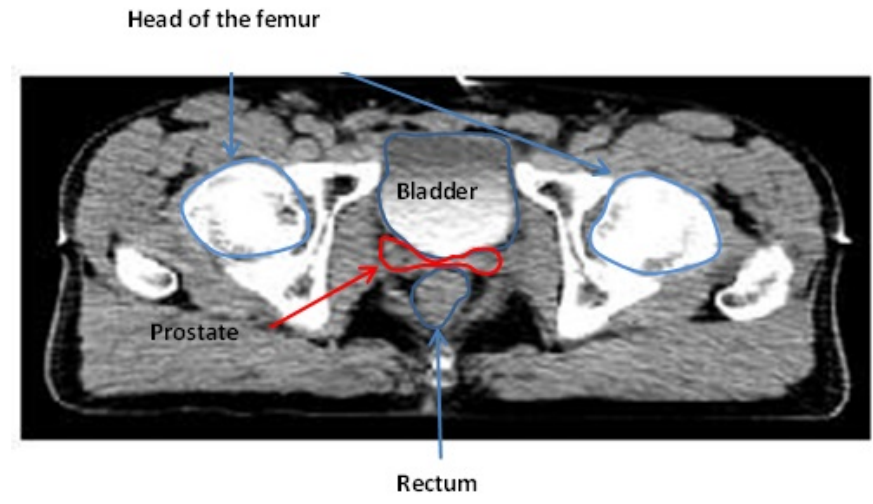

Figure 1. An example of a CT slice

Table 1. The upper limits percentage constraints for organ at risk (QUANTEC)

\begin{tabular}{c|c|c|c}
\cline { 2 - 4 } & \multicolumn{3}{|c}{ Organ } \\
\cline { 2 - 4 } & Head of femur & Rectum & Bladder \\
\hline \multirow{3}{*}{ Upper limits percentage } & & V75 $<15 \%$ & V80 $<15 \%$ \\
& \multirow{2}{*}{ V50 $<5 \%$} & V70 $<20 \%$ & V75 $<25 \%$ \\
& & V65 $<25 \%$ & V70 $<35 \%$ \\
& & V60 $<35 \%$ & V65 $<50 \%$ \\
\hline
\end{tabular}

\subsection{Computational Results on $1 D$ instance}

\subsubsection{Experiments using the exact method}

The combined beam angle and dose distribution problem on 1D Instance is first solved by the exact method using IBM ILOG CPLEX version 12.6. Table 2 shows the results of the exact method where the objective function value $\left(Z^{*}\right)$, the computational time required to solve the problem (CPU) in seconds and the optimal selected beams (beam angle) are presented. For $\eta=4-7$ beams, CPLEX was not able to solve the problem optimally due to out of memory after running over 10 hours. Therefore in this subsection we only analyse the optimal solution when $\eta=4$.

\begin{tabular}{c|c|c|c}
\multicolumn{4}{|c}{ Table 2. Results of the exact method on 1D Instance } \\
\hline$\eta$ & $Z^{*}$ & CPU $(\mathrm{s})$ & Beam angle selected \\
\hline 4 & 115.0239 & 1,512 & $5^{\circ}, 30^{\circ}, 170^{\circ}, 270^{\circ}$ \\
\hline 5 & NA & NA & NA \\
\hline 6 & NA & NA & NA \\
\hline 7 & NA & NA & NA \\
\hline
\end{tabular}

Table 3 presents the average dose that reaches the organ at risk and healthy structures when $\eta=4$. The results also show that each structure has an acceptable percentage of total pixels that receive the dose more than its limit given in Table 1. For example, only $2.69 \%$ of bladder region receives more than 


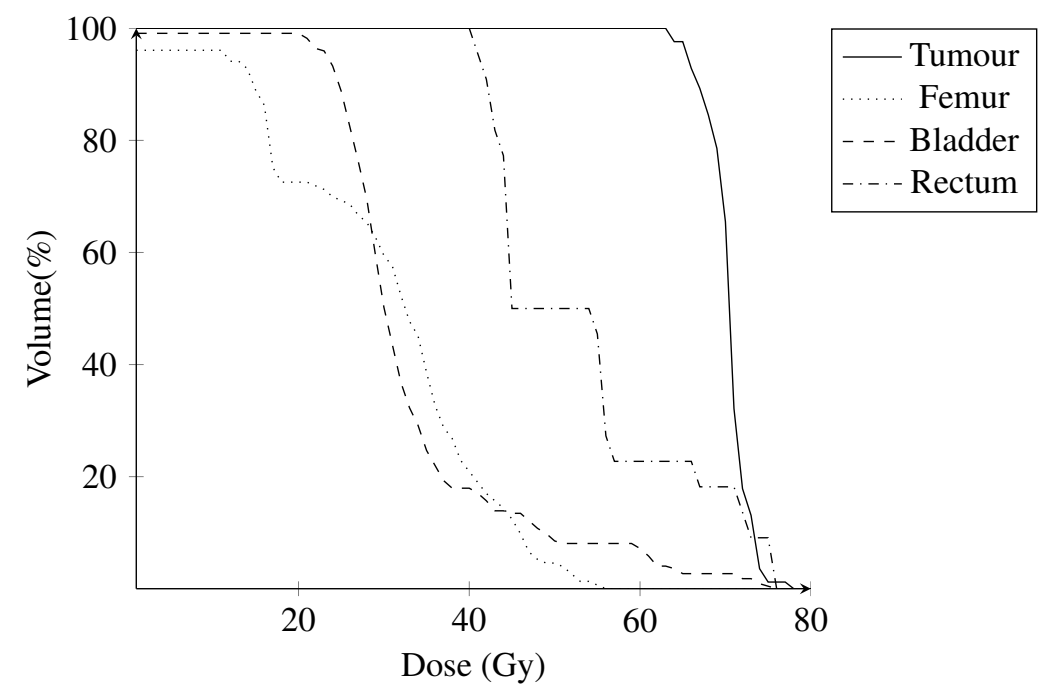

Figure 2: Dose and volume histogram of the optimal solution to 1D Instance with $\eta=4$

$80 \mathrm{~Gy}$ where Table 1 indicates that up to $15 \%$ of bladder region could receive more than $80 \mathrm{~Gy}$. Although the proposed model does not explicitly optimise the percentage of a region that receives the dose, it can be noted that the radiotherapy planning obtained from the optimal solution is very good as the dose covers the tumour structure while preventing significant damage to the organ at risk.

\begin{tabular}{|c|c|c|c|c|c|c|c|c|c|c|c|}
\hline \multirow{2}{*}{$\begin{array}{l}\text { Dose } \\
\text { (Gy) }\end{array}$} & \multicolumn{2}{|c|}{ Femur } & \multicolumn{4}{|c|}{ Bladder } & \multicolumn{5}{|c|}{ Rectum } \\
\hline & Average & Standard & \multicolumn{2}{|c|}{ Average } & \multicolumn{2}{|c|}{$\begin{array}{l}\text { Standard } \\
\text { Deviation }\end{array}$} & & Average & & \multicolumn{2}{|c|}{$\begin{array}{l}\text { Standard } \\
\text { Deviation }\end{array}$} \\
\hline $\begin{array}{l}\text { Dose } \\
\text { limit (Gy) }\end{array}$ & \multicolumn{2}{|c|}{50} & 80 & 75 & 70 & 65 & 75 & 70 & 65 & 60 & 50 \\
\hline $\begin{array}{l}\text { \% pixel } \\
\text { that re- } \\
\text { ceives } \\
\text { more than } \\
\text { the dose } \\
\text { limit }\end{array}$ & \multicolumn{2}{|c|}{4.57} & 0.00 & 0.44 & 2.69 & 2.69 & 9.09 & 18.18 & 22.73 & 22.73 & 50 \\
\hline
\end{tabular}

Figure 2 presents the dose vs volume histogram (DVH), which indicates the dose decomposition per percentage on each organ at risk and tumor. For example: $100 \%$ of the tumor receives at least $63 \mathrm{~Gy}$ and then the percentage decreases with the amount of dose. The optimal solution found here will be used to evaluate the performance of TSrad for solving the combined beam angle and dose distribution problems in radiotherapy planning on Instance 1 with $\eta=4$ beams. 


\subsubsection{Experiments using the matheuristic}

This subsection presents the comparative analysis of the proposed solution method for solving the radiotherapy problem on Instance 1. Table 4 shows the summary of the average, best and worst results of TSrad, where the CPU time and objective function value are also given for the problems with 4-7 beams, respectively. In Table 4, \%gap is provided to determine the deviation of the solution obtained by the corresponding proposed TSrad to the best solution attained from either the exact method or the proposed matheuristic. This \%gap is calculated as follows:

$$
\% \text { gap }=\frac{Z_{m}-Z_{b}}{Z_{m}} \times 100
$$

where $Z_{m}$ and $Z_{b}$ refer to the feasible solution cost obtained by the corresponding matheuristic approach and the best solution respectively. For $\eta=4$, as the optimal solution was obtained by the exact method, the proposed matheuristic will produce an optimal solution if $\% g a p=0$.

For the proposed matheuristic TSrad, preliminary experiments were conducted to determine the parameters values that will be used. Two kinds of neighbourhood are considered, namely deterministic and probabilistic neighbourhoods. For the deterministic neighbourhood, all possible solution neighbours are considered while for the deterministic one, only 10 solution neighbours out of all possible ones are randomly chosen. For both type of neighbourhoods, the value of Tabu tenure is set to 2 . Two experimental scenarios are carried out based on computational time $\left(t_{\max }\right)$. The algorithm will stop when the computing time reached the maximum computational time limit, which is 60 and 1512 seconds, respectively. The first computational time is based on our preliminary experiments where relatively good solutions were found within this short computational time. The second one is based on the computational time required by the exact method to solve the 4 beams problem to optimality. To evaluate the consistency of the proposed matheuristic method, in each problem TSrad is executed 10 times, therefore the average, best and worst results are given.

According to Table 4, TSrad is able to obtain good solutions in a relatively small computing time for the problem with $\eta=4$ beams, and it produces the optimal solution within 60 seconds. When the probabilistic TSrad is executed for 1512 seconds, it obtains the optimal solution for each run as shown by \%gap of the worst result. For the problem with $\eta=5-7$ beams, in general TSrad with probabilistic approach also produces better results than the one with deterministic. The quality of the solution increases with the computational time.

For illustration, Table 5 presents the results on organ at risk and healthy tissues with $\eta=6$ beams where, for all tissues, the percentage of pixels that receive higher dose than the limit is less than the required percentage presented in Table 1. The best solution found is considered as a good solution as the dose also covers the tumour structure while avoiding significant damage on the organ at risk.

Figure 3 presents the dose vs volume histogram (DVH) for the organ at risk and tumour when $\eta$ value is set to 6 . It is worth noting that the diagram for 6 beams produced by TSrad is relatively similar to the one for 4 beam generated by the exact method (CPLEX). 
11 of 17

Table 4. Comparative analysis for TSrad on 1D Instance

\begin{tabular}{|c|c|c|c|c|c|c|c|c|}
\hline \multirow{2}{*}{$\eta$} & \multirow{2}{*}{$\begin{array}{l}t^{\max } \\
(\mathrm{s})\end{array}$} & \multirow{2}{*}{ Neighbourhood } & \multicolumn{2}{|c|}{ Average result } & \multicolumn{2}{|c|}{ Best result } & \multicolumn{2}{|c|}{ Worst result } \\
\hline & & & $Z$ & \%gap & $Z$ & \%gap & $Z$ & \%gap \\
\hline \multirow{4}{*}{4} & \multirow{2}{*}{60} & Probabilistic & 117.31 & 1.98 & 115.02 & 0.00 & 120.82 & 5.04 \\
\hline & & Deterministic & 124.92 & 8.60 & 118.72 & 3.26 & 137.43 & 19.48 \\
\hline & \multirow{2}{*}{1512} & Probabilistic & 115.02 & 0.00 & 115.02 & 0.00 & 115.02 & 0.00 \\
\hline & & Deterministic & 116.1 & 0.10 & 115.02 & 0.00 & 118.43 & 2.96 \\
\hline \multirow{4}{*}{5} & \multirow{2}{*}{60} & Probabilistic & 108.12 & 5.12 & 102.58 & 0.00 & 110.41 & 7.09 \\
\hline & & Deterministic & 117.95 & 13.03 & 107.61 & 4.67 & 126.70 & 19.03 \\
\hline & \multirow{2}{*}{1512} & Probabilistic & 103.40 & 0.79 & 102.58 & 0.00 & 105.59 & 2.84 \\
\hline & & Deterministic & 107.57 & 4.64 & 102.58 & 0.00 & 112.83 & 9.08 \\
\hline \multirow{4}{*}{6} & \multirow{2}{*}{60} & Probabilistic & 101.75 & 6.21 & 95.48 & 0.05 & 105.2 & 9.29 \\
\hline & & Deterministic & 122.98 & 22.40 & 108.11 & 11.73 & 138.17 & 30.93 \\
\hline & \multirow{2}{*}{1512} & Probabilistic & 95.43 & 0.00 & 95.43 & 0.00 & 95.48 & 0.05 \\
\hline & & Deterministic & 98.29 & 2.91 & 95.43 & 0.00 & 103.72 & 7.99 \\
\hline \multirow{4}{*}{7} & \multirow{2}{*}{60} & Probabilistic & 96.76 & 7.76 & 91.77 & 2.76 & 102.48 & 12.92 \\
\hline & & Deterministic & 121,12 & 26.32 & 106.00 & 15.81 & 136.60 & 34.67 \\
\hline & \multirow{2}{*}{1512} & Probabilistic & 89.37 & 0.15 & 89.24 & 0.00 & 90.50 & 1.40 \\
\hline & & Deterministic & 90.38 & 1.28 & 89.23 & 0.00 & 93.91 & 4.98 \\
\hline
\end{tabular}

Table 5. Results of the best solution for organ at risk and healthy tissues on 1D Instance with $\eta=6$ beams

\begin{tabular}{|c|c|c|c|c|c|c|c|c|c|c|c|}
\hline \multirow{4}{*}{$\begin{array}{l}\text { Dose } \\
\text { (Gy) }\end{array}$} & \multicolumn{2}{|c|}{ Femur } & \multicolumn{4}{|c|}{ Bladder } & \multicolumn{5}{|c|}{ Rectum } \\
\hline & Average & Standard & \multicolumn{2}{|c|}{ Average } & \multirow{2}{*}{\multicolumn{2}{|c|}{$\begin{array}{l}\text { Standard } \\
\text { Deviation }\end{array}$}} & \multirow{2}{*}{\multicolumn{3}{|c|}{ Average }} & \multirow{2}{*}{\multicolumn{2}{|c|}{$\begin{array}{l}\text { Standard } \\
\text { Deviation }\end{array}$}} \\
\hline & & & & & & & & & & & \\
\hline & 27.51 & 15.68 & \multicolumn{2}{|c|}{36.72} & \multicolumn{2}{|c|}{9.60} & \multicolumn{3}{|c|}{52.56} & \multicolumn{2}{|c|}{10.09} \\
\hline $\begin{array}{l}\text { Dose } \\
\text { limit (Gy) }\end{array}$ & \multicolumn{2}{|c|}{50} & 80 & 75 & 70 & 65 & 75 & 70 & 65 & 60 & 50 \\
\hline $\begin{array}{l}\% \text { pixel } \\
\text { that re- } \\
\text { ceives } \\
\text { more than } \\
\text { the dose } \\
\text { limit }\end{array}$ & \multicolumn{2}{|c|}{9.15} & 0.00 & 0.45 & 2.69 & 2.69 & 4.55 & 13.66 & 22.73 & 22.73 & 40.91 \\
\hline
\end{tabular}
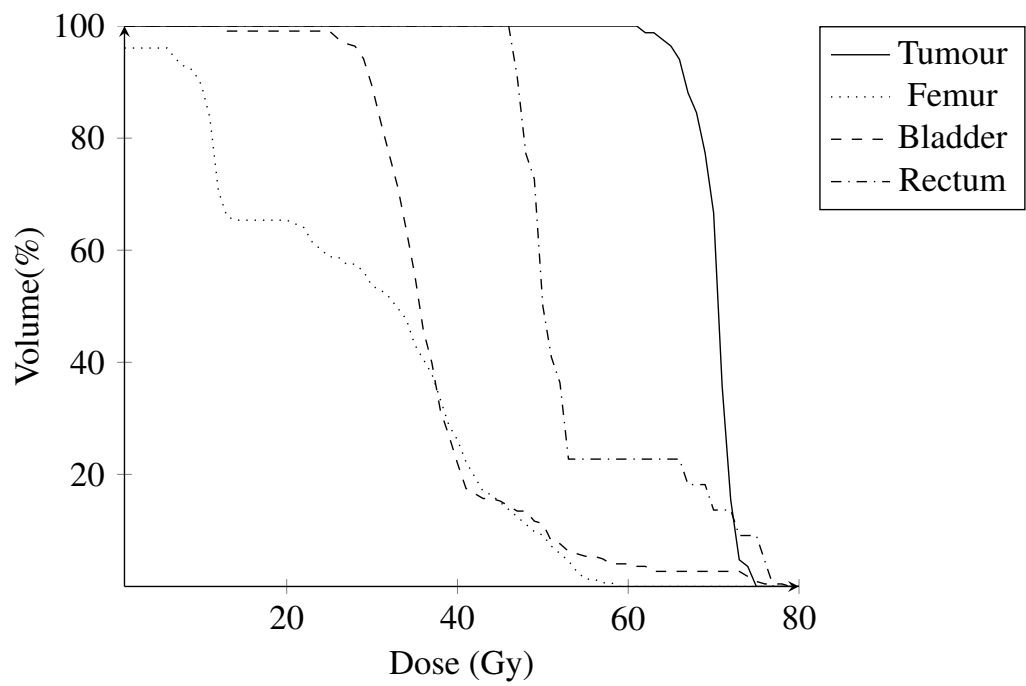

Figure 3: Dose and volume histogram of the best matheuristic solution on 1D Instance with $\eta=6$ beams 


\subsection{Computational Results on 3D instance}

For the experiments on this instance, a set of $30 \mathrm{CT}$ slices is used where the data structure used is the same as the one used in Instance 1. The combined beam angle and dose distribution problems are only solved using TSrad as the exact method is not able to solve them due to memory issues. When a set of selected beams $(X)$ is fixed, the problem reduces to the dose distribution problem expressed in Equations (13)-(17). The dose distribution problem can be divided into $|S|$ sub-problems as each slice has independent objective function and constraints.

In these experiments, the possible angles of the beam are the same as the ones in the previous experiments where there are 72 possible beams available to select. The number of selected beams is also set to 4-7 ( $\eta=4-7)$. Regarding the beam angle problem (solved by TSrad), the complexity of the problem on this instance is the same as the one on Instance 1. The significant challenge is in solving the dose distribution problem (solved by the exact method) within TSrad, as the number of variable and constraints is relatively large. These numbers are dependent on the set of beams which achieves different voxels in each CT slice.

In these experiments, the parameters values of TSrad on 3D instances are set the same as the ones on 1D instance. The maximum computational time $\left(t_{\max }\right)$ is set to 600 and 3,600 seconds. It is set longer than the one in the previous experiments as the computational time required to solve the dose distribution problem is much longer. In the first scenario, the computational time is set to 600 seconds, which is ten times the one in the previous experiments. In the second set of experiments, 3,600 seconds is set which can be considered a realistic upper time limit for providing a viable radiotherapy plan, bearing in mind the volume of patients requiring radiotherapy plans in a hospital. The weights of the objective functions and the dose limits are also set to the same values used for solving 1D Instance. For 3D Instance, the quality of a solution is evaluated by analysing the dose percentage limits and the tumour coverage.

Table 6 presents the average, best and worst result of deterministic and probabilistic TSrad on the 3D Instance with 4-7 beams angles to be selected. Similar to the previous results, probabilistic TSrad outperforms the deterministic one when CPU time is set to 3600 seconds. In the best solution, for $\eta=4$ the selected beam directions are $\left\{55^{\circ}, 180^{\circ}, 265^{\circ}, 330^{\circ}\right\}$ whereas for $\eta=6$ it is $\left\{55^{\circ}, 155^{\circ}, 180^{\circ}, 185^{\circ}, 265^{\circ}, 315^{\circ}\right\}$

For illustration, Tables 7 and 8 resent the average dose that reaches the organ at risk and healthy structures based on the best solution for $\eta=4$ and 6 respectively. Based on the tables, each structure has an acceptable percentage of total pixels that receive the dose more that its limit which is given in Table 1. Figures 4 and 5 describe the dose per percentage in each organ at risk and tumour on 3D instance with $\eta=4$ and 6 respectively.

The analysis on the percentage of voxels that receive the dose based on its limit is considered as an important factor especially in the tumour structure. Lack of doses in this structure will affect the performance of the radiotherapy process. In this sense, although the mathematical model does not include the dose per percentage, the results for both instances are within the imposed limits presented in Table 1. However, there is a relative lack of dose in tumour structure on 3D Instance shown in Figure 4 and 5. Based on the best solution, the percentage of tumour voxels that receive more than or equal to the prescribed dose $70 \mathrm{~Gy}$ are $53 \%$ and $56 \%$ for the 4 and 6 beams problems respectively. The average tumour dose for the 4 and 6 beams problems are $65 \pm 10.78$ Gy and $66.17 \pm 10.34$ Gy respectively. 
Table 6. Comparative analysis for TSrad on 3D Instance

\begin{tabular}{|c|c|c|c|c|c|c|c|c|}
\hline \multirow{2}{*}{$\eta$} & \multirow{2}{*}{$t^{\max }$} & \multirow{2}{*}{ Neighbourhood } & \multicolumn{2}{|c|}{ Average result } & \multicolumn{2}{|c|}{ Best result } & \multicolumn{2}{|c|}{ Worst result } \\
\hline & & & $Z$ & $\%$ gap & $Z$ & $\%$ gap & $Z$ & $\%$ gap \\
\hline \multirow{4}{*}{4} & \multirow{2}{*}{600} & Probabilistic & 3603.85 & 5.93 & 3473.6 & 2.40 & 3805.4 & 10.91 \\
\hline & & Deterministic & 3797.4 & 10.73 & 3446.5 & 1.64 & 4312 & 21.38 \\
\hline & \multirow[b]{2}{*}{3600} & Probabilistic & 3424.97 & 1.02 & 3390.1 & 0.00 & 3474.2 & 2.42 \\
\hline & & Deterministic & 3552.33 & 4.57 & 3426.5 & 1.06 & 3796.1 & 10.70 \\
\hline \multirow{4}{*}{5} & \multirow{2}{*}{600} & Probabilistic & 3458.49 & 6.26 & 3343.2 & 3.02 & 3592.00 & 9.74 \\
\hline & & Deterministic & 3945.14 & 17.82 & 3526.10 & 8.05 & 4770.90 & 32.04 \\
\hline & \multirow{2}{*}{3600} & Probabilistic & 3280.62 & 1.17 & 3242.10 & 0.00 & 3302.00 & 1.81 \\
\hline & & Deterministic & 3617.17 & 10.37 & 3397.60 & 4.58 & 4072.20 & 20.38 \\
\hline \multirow{4}{*}{6} & \multirow[b]{2}{*}{600} & Probabilistic & 3352.91 & 5.71 & 3283.6 & 3.72 & 3510.1 & 9.93 \\
\hline & & Deterministic & 3616.89 & 12.59 & 3323.2 & 4.87 & 4107.3 & 23.03 \\
\hline & \multirow[b]{2}{*}{3600} & Probabilistic & 3190.95 & 0.93 & 3161.4 & 0.00 & 3237.6 & 2.35 \\
\hline & & Deterministic & 3561.75 & 11.24 & 3374.3 & 6.31 & 4003.5 & 21.03 \\
\hline \multirow{4}{*}{7} & \multirow{2}{*}{600} & Probabilistic & 3288.01 & 6.34 & 3179.40 & 3.14 & 3499.30 & 12.00 \\
\hline & & Deterministic & 3520.75 & 12.53 & 3235.80 & 4.83 & 4110.40 & 25.08 \\
\hline & \multirow{2}{*}{3600} & Probabilistic & 3104.89 & 0.82 & 3079.50 & 0.00 & 3133.00 & 1.71 \\
\hline & & Deterministic & 3382.21 & 8.95 & 3208.40 & 4.02 & 3685.60 & 16.45 \\
\hline
\end{tabular}

Table 7. Results of the best solution for organ at risk and healthy tissues on 3D Instance with $\eta=4$ beams

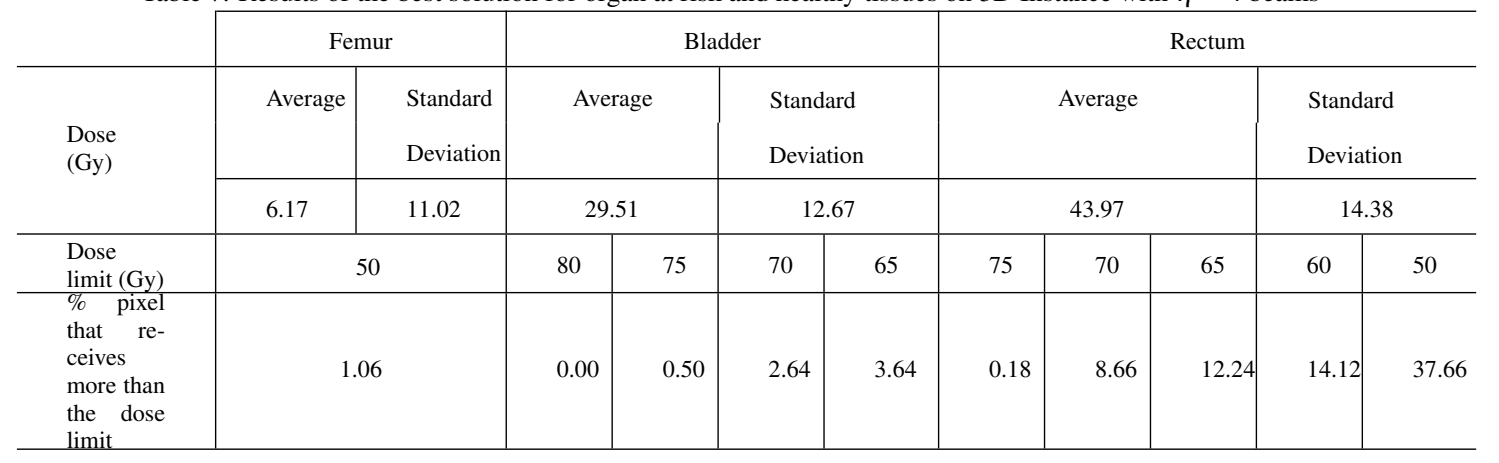

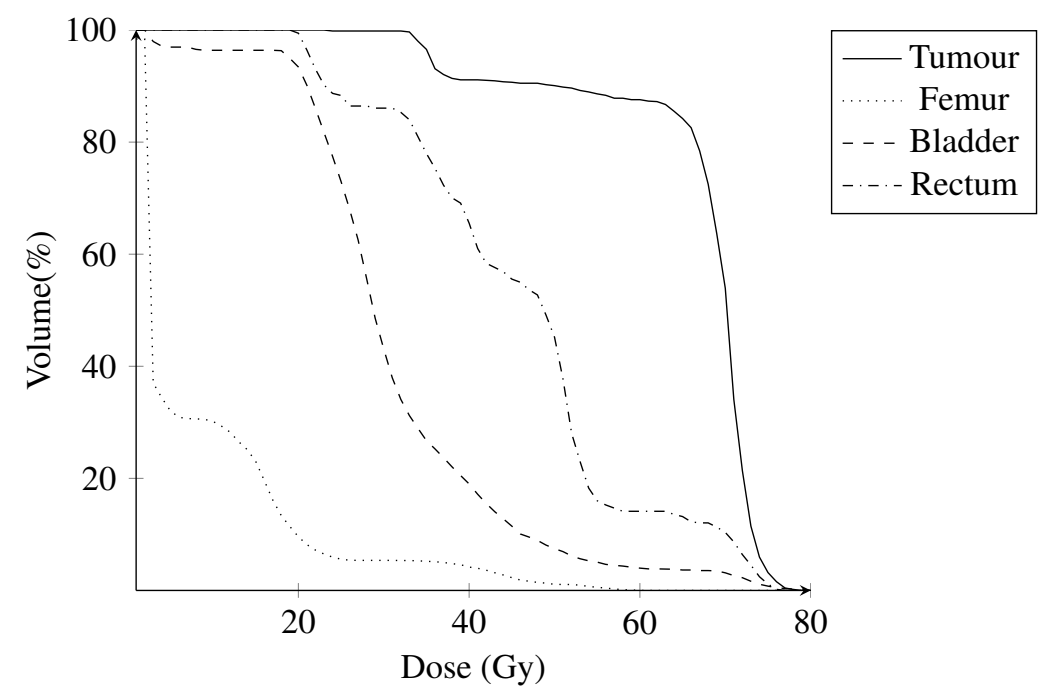

Figure 4: Dose and volume of the best solution on 3D Instance with $\eta=4$ beams 
Table 8. Results of the best solution for organ at risk and healthy tissues on 3D Instance with $\eta=6$ beams

\begin{tabular}{|c|c|c|c|c|c|c|c|c|c|c|c|}
\hline \multirow{2}{*}{$\begin{array}{l}\text { Dose } \\
\text { (Gy) }\end{array}$} & \multicolumn{2}{|c|}{ Femur } & \multicolumn{4}{|c|}{ Bladder } & \multicolumn{5}{|c|}{ Rectum } \\
\hline & Average & Standard & \multicolumn{2}{|c|}{ Average } & \multicolumn{2}{|c|}{$\begin{array}{l}\text { Standard } \\
\text { Deviation }\end{array}$} & \multicolumn{2}{|c|}{ Average } & & \multicolumn{2}{|c|}{$\begin{array}{l}\text { Standard } \\
\text { Deviation }\end{array}$} \\
\hline $\begin{array}{l}\text { Dose } \\
\text { limit (Gy) }\end{array}$ & & & 80 & 75 & 70 & 65 & 75 & 70 & 65 & 60 & 50 \\
\hline $\begin{array}{l}\% \text { pixel } \\
\text { that re- } \\
\text { ceives } \\
\text { more than } \\
\text { the dose } \\
\text { limit }\end{array}$ & \multicolumn{2}{|c|}{0.18} & 0.00 & 0.20 & 1.97 & 3.25 & 0.56 & 5.84 & 13.75 & 16.76 & 29.38 \\
\hline
\end{tabular}

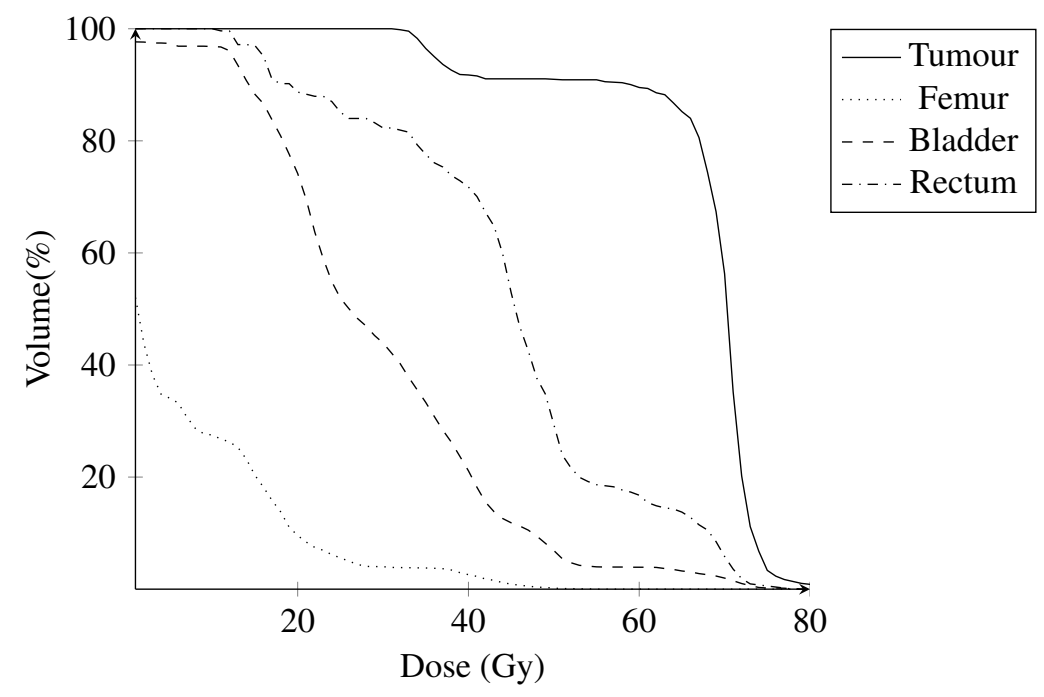

Figure 5: Dose and volume of the best solution on 3D Instance with $\eta=6$ beams

\section{Experiments using different weights}

The weights of the objective functions are affected by several factors such as the singularity of tumor, patient structure and the necessity of custom treatment planning. In this subsection, we also carried out computational experiments using a different weight for each objective function. As the weight represents the importance of the dose deviation for a tissue (tumour, healthy and noble organs), we aim to generate an alternative solution when we change the priority of each objective function. For demonstration purposes, the experiment was performed only on 3D instance with $\eta=4$ using the Probabilistic TSrad as this method yields the best solution in the previous experiments. Here, the weight values are set to as follows: $\gamma_{1}=0.3, \gamma_{2}=0.6, \alpha=\beta=0.05$, which means that we are relatively more concerned about the lack of dose on the tumor organ $\left(\gamma_{2}=0.6\right)$ than the other goals. The excess of dose 
on tumor $\left(\gamma_{1}=0.3\right)$ is the second most important factor followed by the excess of dose on organs at risk and healthy tissues $(\alpha=\beta=0.05)$.

Figure 6 describes the dose per percentage in each organ at risk and tumour on 3D instance with $\eta=4$ beams using a different weight for each objective function. The configuration of the selected beam directions are $\left\{150^{\circ}, 180^{\circ}, 220^{\circ}, 240^{\circ}\right\}$ with the objective function value $(Z)=3,465.15 \mathrm{~Gy}$.

This solution can be considered as a better solution compared to the previous solution as it covers the tumor organs more. However, Table 9 shows that the percentage of dose received by rectum is above the limit (55.41\% of rectum receives more than 50Gy whereas the upper limit is only 50\% as shown in Table 1). Therefore, there is a trade-off/conflict on radiotherapy planning when determining the weight for each objective function. This decision is reliant on the specialist, because it will be patient specific.

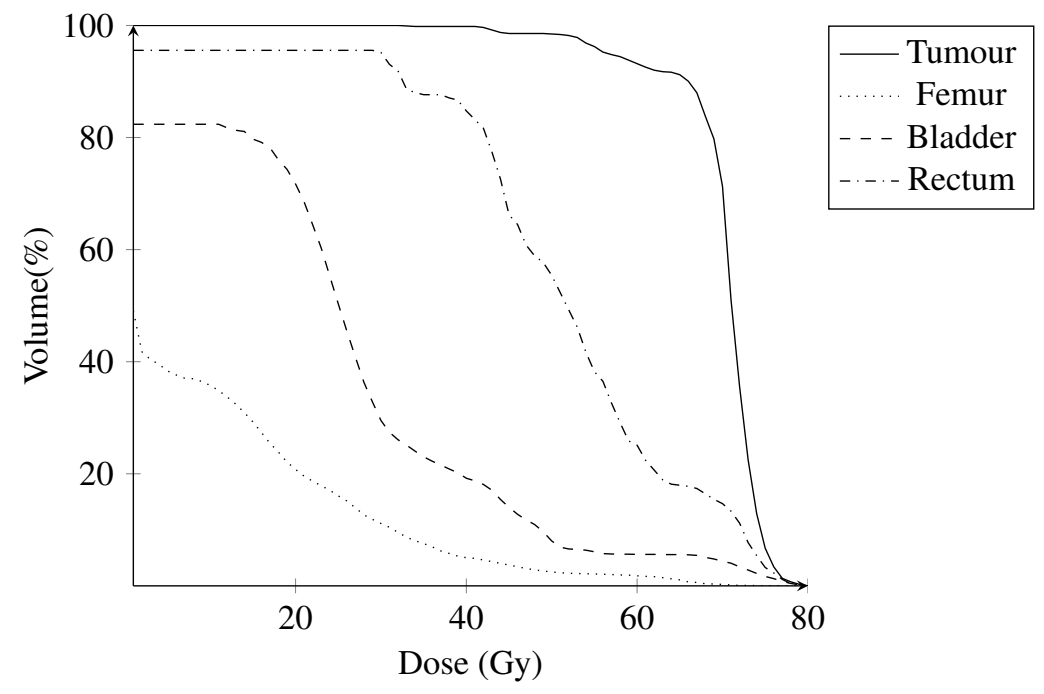

Figure 6: Dose and volume using a different set of weights on 3D Instance with $\eta=4$ beams

Table 9. Results of the TSrad for organ at risk and healthy tissues using a different set of weights

\begin{tabular}{|c|c|c|c|c|c|c|c|c|c|c|c|}
\hline \multirow{2}{*}{$\begin{array}{l}\text { Dose } \\
\text { (Gy) }\end{array}$} & \multicolumn{2}{|c|}{ Femur } & \multicolumn{4}{|c|}{ Bladder } & \multicolumn{5}{|c|}{ Rectum } \\
\hline & Average & Standard & \multicolumn{2}{|c|}{ Average } & \multicolumn{2}{|c|}{$\begin{array}{l}\text { Standard } \\
\text { Deviation }\end{array}$} & \multicolumn{2}{|c|}{ Average } & & \multicolumn{2}{|c|}{$\begin{array}{l}\text { Standard } \\
\text { Deviation }\end{array}$} \\
\hline $\begin{array}{l}\text { Dose } \\
\text { Jimit(Gy) }\end{array}$ & & & 80 & 75 & 70 & 65 & 75 & 70 & 65 & 60 & 50 \\
\hline $\begin{array}{l}\% \text { pixel } \\
\text { that re- } \\
\text { ceives } \\
\text { more than } \\
\text { the dose } \\
\text { limit }\end{array}$ & \multicolumn{2}{|c|}{2.47} & 0.00 & 1.68 & 4.40 & 5.58 & 3.28 & 14.67 & 17.95 & 25.10 & 55.41 \\
\hline
\end{tabular}




\section{Conclusion}

This paper presents a multi-objective optimisation model for the combined beam angle and dose distribution problem. A solution method based on matheuristic technique to tackle large-scale realistic problem which we refer to as TSrad is proposed. A set of experiments were carried out on two instances to evaluate the performance of the proposed matheuristic. The first instance considers one CT slice which can be solved using an exact method when $\eta=4$. Therefore, the performance of the proposed matheuristic can be assessed by comparing their solutions to the optimal one. For this instance, the Probabilistic TSrad produces the optimal solution within 60 seconds. The second instance consists of a set of CT slices which is more realistic in practice than the first one. The proposed matheuristic can solve this instance in a reasonable computational time for the domain of radiotherapy planning. The experiments show that the Probabilistic TSrad has great potential to assist the radiotherapy planning since it provides better solutions for both computational time limits used.

For future work, it is worth investigating the radiotherapy planning problem with non-coplanar beams with CT 3D configuration. Other multi-objective methods, such as Pareto frontier generation and compromise programming, could also be used to produce multiple solutions for the combined beam angle and dose distribution problems.

\section{REFERENCES}

ACOSTA R.; BRICK W.; HANNA A.; HOLDER A.; LARA D.; MCQUILEN G.; NEVIN D.; UHLIG P.; SALTER B.(2009) Radiotherapy optimAl Design: An Academic Radiotherapy Treatment Design System. Operations Research/Computer Science Interfaces, 47 401-425.

BAHR G. K.; KEREIAKES J. G.; HORWITZ H.; FINNEY R.; GALVIN J.; GOODE K. (1968) The method of linear programming applied to radiation treatment planning. Radiology, 91 686-693.

BERTSIMAS D.; CACCHIANI V.; CRAFT D.; NOHADANI O. (2013) A hybrid approach to beam angle optimization in intensity-modulated radiation therapy. Computers \& Operations Research 40 (9) 2187-2197.

BREEDVELD, S. (2013) Towards Automated Treatment Planning in Radiotherapy: A Mathematical Approach to Automated and Integrated Multi-Criterial Optimization of Beam Angles and IMRT Fluence Profiles. Erasmus University Rotterdam, The Netherlands.

CABRERA G. G.; EHRGOTT M.; MASON A. J.; RAITH A. (2018a) A matheuristic approach to solve the multiobjective beam angle optimization problem in intensity-modulated radiation therapy. International Transactions in Operational Research. 25 243-268.

CABRERA-GUERRERO G.; MASON A. J.; RAITH A.; EHRGOTT M. (2018b) Pareto local search algorithms for the multi-objective beam angle optimisation problem. Journal of Heuristics 24 (2) 205-238.

DIAS J.; ROCHA H.; FERREIRA B.; LOPES M. C. (2014) A genetic algorithm with neural network fitness function evaluation for IMRT beam angle optimization. Central European Journal of Operations Research 22 (3) 431-455.

DIAS J.; ROCHA H.; FERREIRA B.; LOPES M. C. (2015) Simulated annealing applied to IMRT beam angle optimization: A computational study. Physica Medica 31 (7) 747-756.

DJENIC A.; MARIC M.; STANIMIROVIC, Z.; STANOJEVIC, P. (2017) A variable neighbourhood search method for solving the long-term care facility location problem. IMA Journal of Management Mathematics 28, (2) 321338.

EHRGOTT M.; HOLDER A.; REESE J. (2008) Beam selection in radiotherapy design. Linear Algebra and its Applications 428 1272-1312.

GEVERT V. G.; OBAL, T. M.; VOLPI, N. M. P., WILHELM, V. E. (2013) [portuguese] Um modelo de otimização integrado para os problemas da escolha de feixes de radiação e do mapa de fluência em radioterapia. In: SIMPÓSIO BRASILEIRO DE PESQUISA OPERACIONAL, XLV, 2013, Natal/RN. Anais... Sociedade Brasileira de Pesquisa Operacional. 
GLOVER F. (1989) Tabu search - Part I. ORSA Journal on Computing 1 190-206.

The continuous single source location problem with capacity and zone-dependent fixed cost: Models and solution approaches. European Journal of Operational Research 263 94-107.

Solving the bi-objective capacitated p-median problem with multilevel capacities using compromise programming and VNS. International Transactions in Operational Research Published online: 1 December 2017. DOI: 10.1111/itor.12485.

Matheuristics: Hybridizing Metaheuristics and Mathematical Programming. Springer, Berlin.

JONES D.; TAMIZ, M. (2010) Pratical Goal Programming. International Series in Operations Research \& Management Science 141 Springer.

LIM, G., CHOIN, J.; MOHAN, R. (2008) Iterative solution methods for beam angle and fluence map optimization in intensity modulated radiation therapy planning. OR Spectrum 30 289-309.

LIM, G. J., CAO W. (2012) A two-phase method for selecting IMRT treatment beam angles: Branch-and-Prune and local neighborhood search. European Journal of Operational Research 217 (3) 609-618.

MARKS, L. B.; TEN, HANKEN, R. K., MARTEL, M. K. (2010) Guest editor: introduction to QUANTEC: a users guide. International Journal of Radiation Oncology Biology Physics 76 (3) S1-S2.

OBAL, T. M.; VOLPI, N. P.; MILOCA, S. A. (2013) Multiobjective approach in plans for treatment of cancer by radiotherapy. Pesquisa Operacional 33 (2) 269-282.

OBAL, T. M.; FLORENTINO, H. O.; GEVERT, V. G.; JONES, D. F.; OUELHADJ, D.; VOLPI, N. M. P.; WILHELM, V. E. (2015) A matheuristic for the selection of beam directions and dose distribution in Radiotherapy Planning. Journal of Physics: Conference Series 616.

PETROVIC, S.; KHUSSAINOVA, G.; JAGANNATHAN, R. (2016) Knowledge-light adaptation approaches in case-based reasoning for radiotherapy treatment planning. Artificial Intelligence in Medicine 68 17-28.

ROCHA, H.; DIAS, J. M.; FERREIRA, B. C.; LOPES, M. C. (2013) Selection of intensity modulated radiation therapy treatment beam directions using radial basis functions within a patter search methods framework. Journal of Global Optimization 57 1065-1089.

ROMEIJN, H. E.; AHUJA, R. K.; DEMPSEY, J. F.; KUMAR, A. (2006) A New Linear Programming Approach to Radiation Therapy Treatment Planning Problems. Operations Research 54 (2) 201-216.

SOUZA, W. D.; MEYER, R. R.; SHI, L. (2004) Selection of beam orientations in intensity-modulated radiation therapy using single-beam indices and integer programming. Physics in Medicine and Biology 49 (15) 346581.

TALBI E G. (2009) Metaheuristics: from desing to implementation. John Wley \& Sons, New Jersey.

VIANA, R. S.; FLORENTINO, H. O.; LIMA, E. A. B. F.; FONSECA, P. R.; HOMEM, T. P. D. (2011) Heterogeneity correction in the construction of optimized planning in radiotherapy using linear programming. Pesquisa Operacional 31 (3) 565-578.

VOET, P. W. J. (2014) Automation of contouring and planning in radiotherapy. Erasmus University Rotterdam, The Netherlands.

XIAO L.; DRIDI M.; EL HASSANI A. H.; LIN W.; FEI H. (2018) A solution method for treatment scheduling in rehabilitation hospitals with real-life requirements. IMA Journal of Management Mathematics, 00, 1-20. 